\title{
Caracterización cromosómica y morfométrica de Carollia perspicillata (Linnaeus 1758) en la región Caribe colombiano
}

\section{Chromosomal and morphometric characterization of Carollia perspicillata (Linnaeus 1758) in Colombian Caribbean region}

\section{Margarita Rumié Mendoza ${ }^{1}$, Julio Chacón Pacheco ${ }^{2,3}$ y Manolo Jaramillo García ${ }^{1 *}$}

\author{
1 Grupo de Investigación Biomédica y Biología Molecular, Facultad de Ciencias de la Salud, Universidad del Sinú. Cra. 1W No. 38-153 Barrio Juan XXIII, \\ Montería, Córdoba, Colombia. Código postal 230001. \\ 2 Grupo de Biodiversidad Unicórdoba, Facultad de Ciencias Básicas, Departamento de Biología. Universidad de Córdoba, Carrera 6 núm. 76-103, Montería \\ Córdoba, Colombia. Código postal 230001 \\ 3 Grupo de Investigación AMDAC, Institución Educativa José María Córdoba, calle 29 Na 16b-43 Barrio San José, Montería Córdoba, Colombia. \\ * Autor para correspondencia \\ E-mail Margarita Rumié: margarita.rumie@gmail.com \\ Email Julio Chacón: jchacon_bio@hotmail.com \\ Email Manolo Jaramillo: mjaramillog@gmail.com
}

\section{Resumen}

El género Carollia es un grupo diverso de murciélagos neotropicales. La similitud de caracteres morfométricos y alto grado de diversidad, dificulta la identificación precisa de las especies del género. Pese a ser un género común en Colombia, no existen reportes citogenéticos para la región Caribe, por tanto, se caracterizó su cariotipo y morfometría craneal, aportando datos citogenéticos útiles para dilucidar la taxonomía del género. Se realizaron muestreos en fragmentos de bosque y reservas de la sociedad civil del departamento de Córdoba. Se capturaron 15 individuos (6 machos, 9 hembras) obteniendo valores promedios de antebrazo de 41.92 mm, tibia de $18.49 \mathrm{~mm}$ y peso de $16.80 \mathrm{~g}$, además de los valores obtenidos para las medidas craneales, dentales y mandibulares que confirman que el material fue correctamente catalogado como C. perspicillata. Cinco ejemplares fueron cariotipados (3 machos, 2 hembras), contabilizando 262 metafases. El número diploide de $C$. perspicillata se determinó de $2 n=20 / X X$ para hembras y $2 n=21 X Y, Y_{2}$ para machos con Número Fundamental = 36. Por bandeo G se encontró homogeneidad en los pares 1, 2, 3 y 5. Con este trabajo, se incrementa la representatividad geográfica de los estudios citogenéticos de $C$. perspicillata para la costa norte de Colombia, conservando normalidad en cuanto al número cromosómico reportado.

Palabras clave: bandas G; citogenética; Chiroptera; morfometría craneal.

\section{Abstract}

The Carollia genus is a diverse group of bats from the Neotropical region, the similarity of its morphometric characters and high level diversity make it difficult to pinpoint the exact identification of species from this genus along its distribution. However, despite the Carollia being common for Colombia, there does not exist some cytogenetic research for Colombian Caribbean region, therefore, it characterized its karyotype and cranial morphometry, providing useful data that can contribute to taxonomic identification of the genus. The specimens of $C$. perspicillata samples were collected in forest fragments and civil society reserves in the Córdoba department, Colombia. Captured 15 individuals ( 6 males and 9 females) obtaining average forearm values of $41.92 \mathrm{~mm}$, tibia $18.49 \mathrm{~mm}$ and weight $16.80 \mathrm{~g}$, in addition to the values obtained for the cranial, dental and mandibular measurements that confirm that the material was catalogued correctly as C. perspicillata. Five specimens were used for the karyotype analysis ( 3 males, 2 females), counting 262 metaphases. The diploid number of $C$. perspicillata was determined from $2 n=20 / X X$ for females and $2 n=21 X Y 1 Y 2$ for males with Fundamental Number $=36$. By band G, homogeneity was found in pairs 1,2,3 and 5. This research increases the geographic representativeness of the cytogenetic studies of $C$. perspicillata for the northern of Colombia, preserving normality in terms of the chromosomal number reported.

Keywords: G-bands; Chiroptera; cytogenetic; skull morphometry.

\section{Citación:}

Rumié Mendoza M., J. Chacón Pacheco \& M. Jaramillo García. 2018 Caracterización cromosómica y morfométrica de Carollia perspicillata (Linnaeus 1758) en la región Caribe colombiano. Revista peruana de biología 25(4): 417 - 424 (Diciembre 2018). doi: http://dx.doi.org/10.15381/ rpb.v25i4.15533

$\begin{array}{ll}\text { Presentado: } & 05 / 07 / 2017 \\ \text { Aceptado: } & 02 / 08 / 2018\end{array}$

Publicado online: 07/12/2018
Información sobre los autores:

MRM: Revisión bibliográfica, captura de especímenes, estandarización de protocolos citogenéticos, análisis cariotípico, procesamiento de datos morfométricos, elaboración del primer borrador del artículo. JCP: Captura e identificación de especímenes, toma de datos morfométricos, análisis de datos morfométricos, revisión de la versión final del manuscrito. MJG: Concepción de la idea, revisión bibliográfica, estandarización de protocolos citogenéticos, análisis cariotípico, elaboración de la versión final. Los autores declaran no incurrir en conflictos de intereses. 


\section{Introducción}

Los murciélagos constituyen el segundo orden taxonómico más diverso entre los mamíferos en el mundo y en Colombia. Su diversidad y abundancia los ha convertido en componentes importantes de la fauna tropical, ademas de sus papeles como polinizadores, dispersores de semillas y depredadores de artrópodos, entre otros (Fleming 1982). Son un grupo de gran importancia en la evaluación de los hábitats y su fragmentación, son capaces de volar sobre áreas extensas y ecológicamente diversas (Medellín et al. 2000).

El grupo está conformado por más de 1100 especies a nivel mundial (Simmons 2005) y 220 especies en el Neotrópico (Gardner 2008). Colombia es considerado el segundo país con más especies de murciélagos en el mundo y el primero en América con 205 especies registradas (Ramírez-Chaves \& Noguera 2010), de este número de especies, en el departamento de Córdoba han sido identificadas ocho familias, 36 géneros y 53 especies (Ballesteros \& Racero-Casarrubia 2012, RaceroCasarrubia et al. 2015, Vela-Vargas \& Pérez-Torres 2012). Siendo, la familia Phyllostomidae la que presenta el mayor número de especies, representada por 35 especies (Racero-Casarrubia et al. 2015). Los murciélagos pertenecientes a esta familia, representan un linaje marcado por una diversidad morfológica y ecológica sin precedentes, y extensa reorganización cromosómica. Lo que conlleva a que todavía existan desacuerdos en cuanto a sus relaciones sistemáticas debido a la convergencia morfológica entre algunos grupos (Sotero-Caio et al. 2013).

El género Carollia, miembro de Phyllostomidae, representa un grupo diverso de murciélagos de la región neotropical. Su distribución abarca desde México hasta Paraguay (MacLellan \& Koopman 2008). La similitud de caracteres morfométricos, alto grado de diversidad, forma y la superposición de los hábitats naturales han dificultado la identificación precisa de las especies de este género a lo largo de su distribución, lo que actualmente supone un problema taxonómico para este grupo de murciélagos (Solari \& Martínez-Arias 2014). Los miembros del género, juegan un papel clave en la dinámica del bosque, en la dispersión de semillas (aunque también consumen insectos) y en la promoción y regeneración del bosque, contribuyendo al mantenimiento florístico y a la diversidad faunística (Harrison 2005)

Este género ha servido de modelo para varios estudios en diversos campos como la ecología (Muscarella \& Fleming 2007), conservación (Medellín et al. 2000), descripción de la estructura social (Peñuela-Salgado \& Pérez-Torres. 2015) y filogeografía (Hoffmann \& Baker 2003). Sin embargo, estos estudios se han basado en la controversial taxonomía del género, que proporciona una limitada interpretación de la relación de los taxones (Castro-Luna \& Galindo-González 2012, Solari \& Martínez-Arias 2014).

Las especies del género Carollia presentan gran semejanza morfométrica entre sí, lo que se ha constituido como un problema particular al momento de la identificación taxonómica, tanto que algunas especies han sido consideradas sinonimia (Zurc \& Velazco 2010). Es por esto y por la variabilidad genética, que Carollia es considerado un género de interés en la realización de estudios de las características cromosómicas (Baker \& Bradley 2006), lo que a su vez sugiere la realización de análisis cariológicos más estrictos, como lo son el uso de patrones de bandeo cromosómico (Baker 1967; Patton \& Gardner 1971; Stock 1975).

Para Colombia, se han realizado cariotipos en las especies $C$. brevicauda y C. perspicillata de los departamentos de Amazonas y Nariño (Baker \& Bleier 1971, Noguera 2008, Noguera \& Muñoz-Montenegro 2014). Para C. perspicillata se ha reportado un $2 \mathrm{n}$ : 20 para hembras y 2n: 21 para machos y un número fundamental (NF): 36 (Noguera 2008). Sin embargo, pese a ser Carollia un género común para el departamento de Córdoba (Ballesteros \& Racero-Casarrubia 2012, Calonge et al. 2010, Suárez-Villota et al. 2009, Vela-Vargas \& Pérez-Torres 2012), no existe ningún reporte citogenético que contribuya a su identificación taxonómica, situación semejante para la región Caribe. Por lo cual, éste estudio buscó caracterizar cariotípica y morfométricamente la especie $C$. perspicillata para Córdoba y aportar datos citogenéticos útiles en la tarea de dilucidar la taxonomía del género.

\section{Materiales y métodos}

Área de estudio.- Esta investigación se desarrolló en fragmentos de bosque seco (bs-T) y húmedo tropical (bh-T) asociados a sistemas de ganadería extensiva y reservas de la sociedad civil, localizados en el departamento de Córdoba, al noroccidente de Colombia; región de $23980 \mathrm{~km}^{2}$ que representa el $2.1 \%$ del territorio del país. Los ejemplares de C. perspicillata fueron capturados en los municipios de Montería y Tierralta, ubicados en el centro y sur del departamento respectivamente. Las localidades de estudio corresponden a la Estación Ecológica Las Guartinajas $\left(8^{\circ} 03^{\prime} 40.0^{\prime \prime} \mathrm{N}\right.$; 760' $\left.30.0^{\prime \prime} \mathrm{W}\right)$; zona intervenida en proceso de restauración ecológica (Chacón et al. 2015); Finca Tuti Fruti, vereda Tuis Tuis $\left(8^{\circ} 02^{\prime} 36.0^{\prime \prime} \mathrm{N} ; 7^{\circ} 06^{\prime} 01.1^{\prime \prime W}\right)$ y Finca el Danubio ( $\left.8^{\circ} 17^{\prime} 19.6^{\prime \prime} \mathrm{N} ; 7^{\circ} 59^{\prime} 38.7^{\prime \prime} \mathrm{W}\right)$ en el municipio de Tierralta, y la Finca Santa Isabel (8³6 $\left.42.0^{\prime \prime} \mathrm{N} ; 75^{\circ} 44^{\prime} 32.0^{\prime W}\right)$ en el municipio de Montería (Fig. 1).

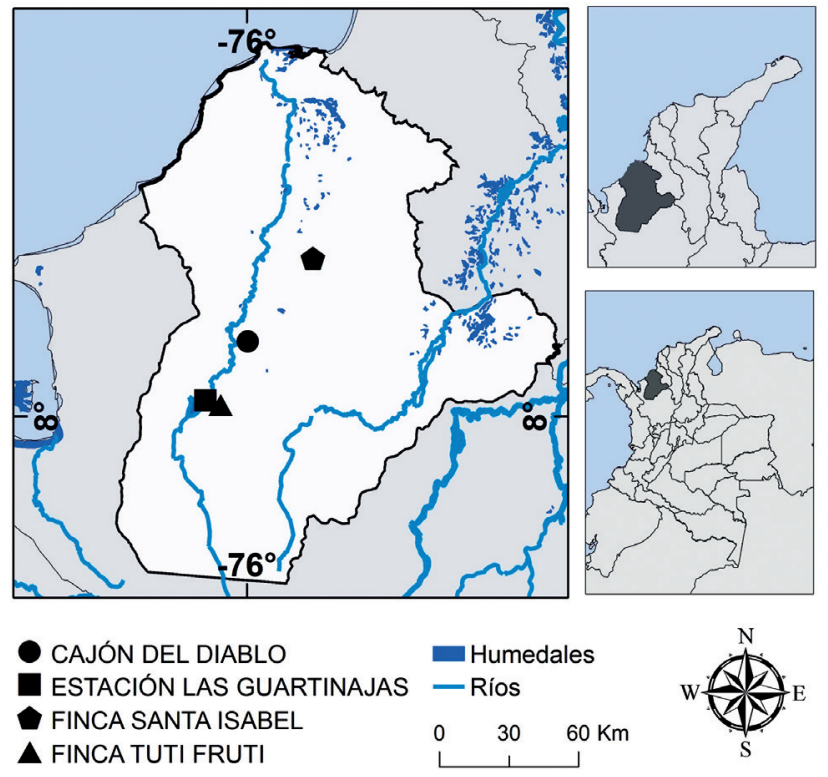

Figura 1. Ubicación de las localidades de estudio en los municipios de Montería y Tierralta, departamento de Córdoba, Colombia. 
Colecta de material biológico.- Los muestreos se realizaron entre agosto de 2015 y marzo de 2016, utilizando cuatro redes de niebla (6x3 metros), ubicadas al interior de fragmentos de bosque y cerca de cuerpos de agua de tal manera que se pudiera maximizar el éxito de la captura de los murciélagos, desde las 18:30 hasta las 00:30, siendo monitoreadas cada 30 minutos. Los murciélagos capturados (machos y hembras) de C. perspicillata se depositaron en bolsas de tela. Posteriormente, se realizó la identificación en campo (Timm \& LaVal 1998, Linares 1998, Gardner 2008), teniendo en cuenta medidas morfométricas de antebrazo $(\mathrm{AB})$ y tibia $(\mathrm{T})$, los cuales fueron tomadas con un calibrador digital $(0.01 \mathrm{~mm})$, se tomó peso del animal, sexo y observación dental de caninos e incisivos inferiores.

Los especímenes fueron colectados, etiquetados y depositados en la Colección de Zoología de la Universidad de Córdoba CZUC. Además, de las medidas morfométricas externas, se tomaron 18 medidas craneales y dentales de gran valor biológico y estadístico que fueron comparadas con los trabajos de Noguera (2008) y Zurc y Velazco (2010). Las medidas craneales, dentales y mandibulares consideradas fueron:

- LMC: Longitud máxima del cráneo; la mayor longitud obtenida en el cráneo, desde la cresta lambdoidea hasta la parte distal de los premaxilares.

- LHMAX: longitud de la hilera dental superior; Distancia lineal entre la proyección distal de la base del canino superior, hasta la proyección distal posterior del premolar tres sobre el mismo lado del cráneo.

- LHDS: Longitud hilera superior antes del canino; Distancia lineal entre la proyección distal del primer premolar superior, hasta la proyección distal posterior del molar tres sobre el mismo lado del cráneo.

- CC: Ancho de los caninos; Es la mayor distancia entre los puntos más externos de los caninos.

- EXM1M1: Ancho exterior M1-M1; Es la mayor distancia entre los puntos más externos de los primeros molares.

- EXM2M2: Ancho exterior M2-M2; Es la mayor distancia entre los puntos más externos de los segundos molares.

- EXM3M3: Ancho exterior M3-M3; es la mayor distancia entre los puntos más externos de los terceros molares.

- INM1M1: Distancia interna M1-M1; Es la distancia entre los puntos más internos de los primeros molares.

- INM2M2: Distancia interna M2-M2; Es la distancia entre los puntos más internos de los segundos molares.

- INM3M3: Distancia interna M3-M3; Es la distancia entre los puntos más internos de los terceros molares.

- INPM2-PM2: Distancia interna PM2-PM2; Es la distancia entre los puntos más internos de los segundos molares.

- DEPM1-PM1: Distancia externa PM1-PM1; Es la distancia entre los puntos más externos de los primeros molares.

- DM3PM2: longitud de la línea de molares más PM2; Es la mayor distancia entre los puntos más externos del tercer molar hasta el extremo distal del premolar dos (PM2) sobre el mismo lado del cráneo.
- PAPM2-PM2: Palatino PM2-PM2; Es la distancia máxima interna entre los puntos de oclusión PM2-PM1.

- PAM3-M3: palatino M3-M3; es la distancia máxima interna entre los puntos de oclusión M3-PM2.

- LMAN: longitud de la mandibular; Desde la mayor proyección del incisivo inferior mandibular hasta la parte distal del ramus mandibular del mismo lado.

- $\quad$ LHMAN: Longitud hilera dental mandíbula; Distancia lineal entre la proyección distal del canino inferior, hasta la proyección distal posterior del molar tres sobre el mismo lado del cráneo.

- LHC: Longitud hilera dental de la mandíbula antes del canino: Distancia lineal entre proyección distal del primer premolar inferior, hasta la proyección distal posterior del molar tres sobre el mismo lado del cráneo.

Obtención de metafases y bandeo cromosómico.- Cinco especímenes ( 2 hembras, 3 machos) fueron procesados para obtención de metafases en campo de acuerdo a lo propuesto por Baker y Qumsiyeh (1988). Las preparaciones cromosómicas fueron realizadas a partir de medula ósea de húmero y tinción estándar con Giemsa (Baker et al. 2003, Noguera-Urbano \& Muñoz-Montenegro 2014), previa aplicación de Colchicina al $0.1 \%$ por espacio de dos horas $(0.1 \mathrm{~mL}$ por cada $10 \mathrm{~g}$ de peso del individuo). Las células de médula ósea fueron sometidas a tratamiento hipotónico con $\mathrm{KCl} 0.075 \mathrm{M}$ durante 90 mínutos, incubadas a calor corporal y fijados en Carnoy fresco (3:1 metanol-ácido acético).

Se determinó el número diploide (2n) mediante conteo de cromosomas en células metafásicas y el número fundamental (NF) identificado como el número total de los brazos de los autosomas. Se tomaron registros fotográficos de metafases para macho y hembra. El cariotipo se organizó de acuerdo a Baker y Bleier (1971), realizando medidas de los brazos cortos (p) y largos (q) con el software IdeoKar 1.2 (http://agri.uok.ac.ir/ideokar/ index.html). Para clasificar los cromosomas se tuvieron en cuenta los términos: metacéntrico, submetacéntrico, subtelocéntrico y acrocéntrico, definidos por Patton (1967); los cromosomas se clasificaron considerando la relación entre brazo largo y corto (RB), índice centromérico (IC) y la longitud relativa (LR) (Levan et al. 1964), todo esto para macho y hembra. Adicionalmente, se presentan datos de longitud de los brazos cortos p y largos q, con lo que se determinaron la relación de brazos RB (l/s), el índice centromérico IC $[(\mathrm{p} / \mathrm{p}+\mathrm{q}) \times 100]$ y la longitud relativa $\mathrm{RL}$ (longitud total del cromosoma $/ \Sigma$ longitud de los cromosomas del conjunto haploide) $\mathrm{x} 100)$.

Para la identificación de características morfológicas de cada cromosoma se realizó la técnica de bandeo G (Jac \& Scheres 1972) con modificaciones, realizando envejecimiento de placas por 8 días. Para el análisis de bandas $\mathrm{G}$ se utilizó solamente el cariotipo de hembra, debido a que no presentan complejidad en sus pares autosómicos y sexuales.

Análisis estadístico.- Se aplicaron estadísticos descriptivos, media, desviación estándar e intervalos de confianza a datos morfométricos, craneales, mandibulares y dentales. Igualmente, se describieron parámetros cariotípicos y cromosómicos como: longitud total de cada cromosoma (CL), medición de brazos $\mathrm{p}$ y q, relación de los brazos (AR) porcentaje de longitud relativa de los cromosomas (RL\%), índice centromérico (IC), r-valor y 
tipo de cromosoma (Type) de los cariotipos de macho y hembra de C. perspicillata.

\section{Resultados}

Se capturaron 15 individuos de la especie C. perspicillata, de los cuales 6 fueron machos y 9 hembras. Se determinó para los individuos una longitud de antebrazo promedio de $41.92 \mathrm{~mm}$; pelaje corto y poco denso; longitud de la tibia promedio de 18,49 $\mathrm{mm}$ y un peso promedio de $16.80 \mathrm{~g}$. Cuando la mandíbula es vista directamente desde la parte anterior, se observaron los incisivos ocultados por la singula de los caninos y/o por la línea superior dental fuera (Tabla 1, Fig. 2).

De los 15 ejemplares capturados, se tomaron cinco individuos de $C$. perspicillata (2 hembras, 3 machos) para realizar la obtención extendidos metafásicos. Se contabilizaron 262 células metafásicas. El número diploide de C. perspicillata para el departamento de Córdoba se determinó de $2 \mathrm{n}=20$ /XX para hembras y $2 \mathrm{n}=21 \mathrm{XY}_{1} \mathrm{Y}_{2}$ para machos con $\mathrm{NF}=36$ (Fig. 3).

A partir de los datos: índice centromérico (IC) y relación de longitud de los brazos (AR); los cromosomas de Carollia perspicillata, están clasificados en tres grupos de cromosomas autosómicos, basados en su tamaño y medida de brazos, de la siguiente manera: metacéntricos $(\mathrm{M})$, submetacéntricos $(\mathrm{SB})$ y acrocéntricos (A). El cromosoma X, por su parte, se clasifica como subtelocéntrico (ST), distinguible por presentar una constricción secundaria ubicada cerca del centrómero muy marcada en los brazos largos. En los individuos machos fue evidente la presencia de los cromosomas $\mathrm{Y}_{1} \mathrm{Y}_{2}$. El cromosoma $\mathrm{Y}_{1}$ es acrocéntrico largo y $\mathrm{Y}_{2}$ es un cromosoma pequeño acrocéntrico, los cuales son organizados en orden decreciente de tamańo (Fig. 4).

Tabla 1. Medidas de los ejemplares de Carollia perspicillata del departamento de Córdoba, Colombia. La media y desviación estándar / entre paréntesis el rango / el número de casos (n).

\begin{tabular}{|c|c|c|c|}
\hline Variables & Este trabajo & Noguera-Urbano (2008) & Zurc y Velasco (2010) \\
\hline TIBIA / (T) & $18.49 \pm 1.41 /(15.18-21.31) / 15$ & & \\
\hline ANTEBRAZO / (AB) & $41.92 \pm 1.46 /(39.72-44.92) / 15$ & $42.2 \pm 1 / 12$ & 41.29 / (39.5 - 44) / 38 \\
\hline PESO / (P) g & $16.80 \pm 1.85 /(14-21.7)$ & & \\
\hline LMC & $22.68 \pm 0.57 /(21.69-23.81) / 15$ & $22.98 \pm 0.58 / 12$ & $22.58 /(21.40-24.16) / 84$ \\
\hline LHMAX & $7.74 \pm 0.20 /(7.46-8.18) / 15$ & $7.92 \pm 0.28 / 12$ & \\
\hline LHDS & $6.31 \pm 0.25 /(5.66-6.74) / 15$ & $6.29 \pm 0.17 / 12$ & $7.94 /(6.83-9.27) / 85$ \\
\hline $\mathrm{CC}$ & $5.02 \pm 0.22 /(4.72-5.3) / 15$ & $5.13 \pm 0.16 / 12$ & $4.90 /(4.49-5.38) / 85$ \\
\hline EXM1M1 & $6.65 \pm 0.26 /(6.06-6.99) / 15$ & $6.38 \pm 0.49 / 12$ & \\
\hline EXM2M2 & $7.46 \pm 0.26 /(6.98-7.76) / 15$ & $7.69 \pm 0.58 / 12$ & \\
\hline EXM3М3 & $8.10 \pm 0.35 /(7.51-8.87) / 15$ & $8.18 \pm 0.15 / 12$ & $8.01 /(7.32-8.81) / 85$ \\
\hline INM1M1 & $3.73 \pm 0.36 /(3.31-4.7) / 15$ & $3.72 \pm 0.2 / 12$ & 3.69 / (3.02-4.08) / 85 \\
\hline INM2M2 & $4.46 \pm 0.51 /(3.84-5.59) / 15$ & $4.27 \pm 0.19 / 12$ & $4.24 /(3.52-4.65) / 84$ \\
\hline INM3M3 & $5.83 \pm 0.29 /(5.38-6.51) / 15$ & $6.11 \pm 0.14 / 12$ & $6.01 /(5.41-6.65) / 85$ \\
\hline INPM2-PM2 & $3.75 \pm 0.25 /(3.32-4.2) / 15$ & $3.81 \pm 0.06 / 12$ & $4.38 /(3.47-5.52) / 85$ \\
\hline DEPM1-PM1 & $5.12 \pm 0.29 /(4.68-5.61) / 15$ & $5.16 \pm 0.16 / 12$ & \\
\hline DM3PM2 & $5.08 \pm 0.41 /(4.56-6.08) / 15$ & $4.88 \pm 0.13 / 12$ & \\
\hline PAPM2-PM2 & $3.88 \pm 0.27 /(3.48-4.49) / 15$ & $4.4 \pm 0.18 / 12$ & \\
\hline PAM3-M3 & $5.88 \pm 0.22 /(5.52-6.35) / 15$ & $6.24 \pm 0.13 / 12$ & \\
\hline LMAN & $14.98 \pm 0.87 /(12.22-15.75) / 15$ & $15.25 \pm 0.39 / 12$ & 14.72 / (13.55 - 17.4) / 85 \\
\hline LHMAN & $8.33 \pm 0.36 /(7.43-8.82) / 15$ & $8.84 \pm 0.2 / 12$ & 8.59 / (7.85 - 9.21) / 85 \\
\hline LHC & $7.34 \pm 0.58 /(6.61-8.83) / 15$ & $7.08 \pm 0.22 / 12$ & \\
\hline
\end{tabular}
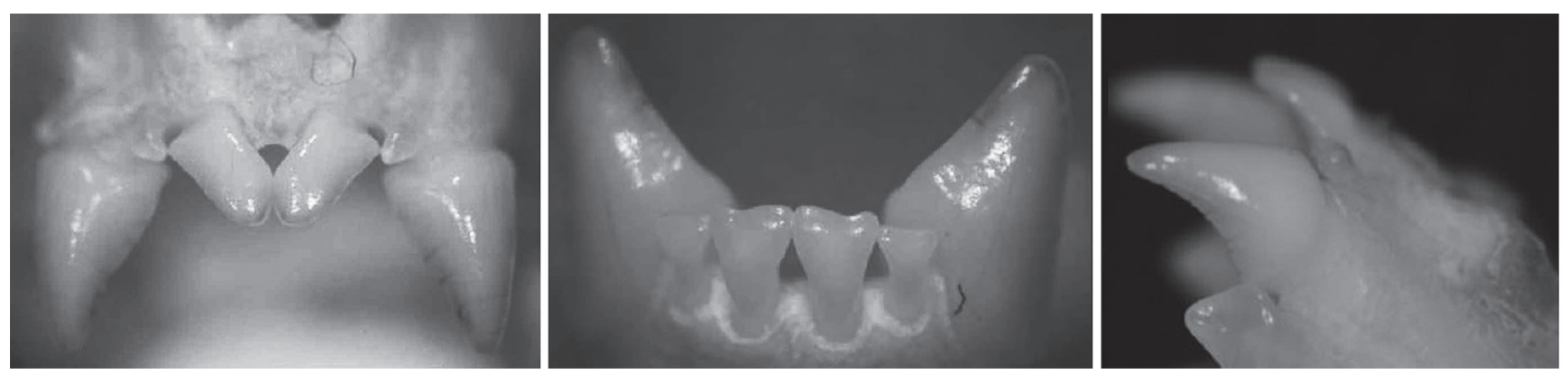

Figura 2. Vista anterior y lateral de caninos e incisivos de Carollia perspicillata colectado en el departamento de Córdoba, Colombia. 
Por otro lado, se determinó el tipo de cromosomas (Type) y la fórmula cariotípica para macho y hembra: Macho: $\mathrm{XY}_{1} \mathrm{Y}_{2}: 2: 2: 5$, donde el grupo 1 (sexual): X (ST), $\mathrm{Y}_{1}(\mathrm{~A}), \mathrm{Y}_{2}(\mathrm{~A})$; Grupo 2: par 3 y 4 (ST); Grupo 3: par 5 y 6 (SM); Grupo 4: par 2, 7, 8, 9, 10 (M). Hembra: 1:1:3:5, donde el grupo 1(sexual ST); Grupo 2: par 3 ST; Grupo 3: par 4,5 y 6 (SM); Grupo 4: par 2, 7, 8, 9, 10.

En el análisis de bandeo cromosómico, se encontró para bandas $G$, homogeneidad en los pares 1, 2, 3 y 5; donde se revelan bandas en las regiones intersticiales del brazo corto del cromosoma 1, y en las regiones teloméricas de los brazos cortos de los pares cromosómicos 2, 3 y 5 (Fig. 5).

\section{Discusión}

Los 15 individuos colectados, pertenecían a material correctamente catalogado de acuerdo a sus medidas craneales y dentales a $C$. perspicillata y se caracterizan por tener similitudes con $C$. monohernandezi, siendo las especies de mayor tamaño del género, pero sus estructuras morfocraneales marcan su diferencia, datos que coinciden con lo descrito por Muñoz et al. (2004) y Zurc y Velazco (2010).

Los resultados obtenidos en este estudio, demuestran que C. perspicillata, conserva normalidad en cuanto al número cromosómico reportado por otros investigadores (Baker 1967, Cloutier \& Thomas 1992, Patton \& Gardner 1971). Para las distintas especies del genero Carollia, argumentan que el número cromosómico es muy estable y se ha logrado mantener así desde hace 7 millones de años aproximadamente, sin embargo, la diferenciación específica ha ocurrido en otras especies del género, provocando grupos polimórficos como las poblaciones de C. benkeithi, pertenecientes al complejo C. castanea (Solari \& Baker 2006).

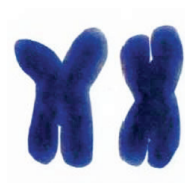

2

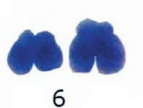

6

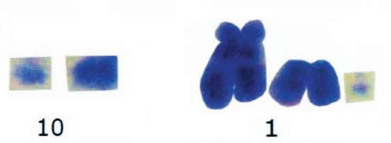

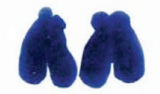

4

5

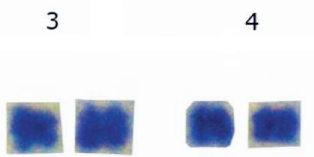

8

(A)

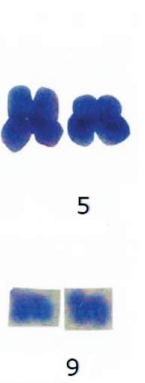

X8
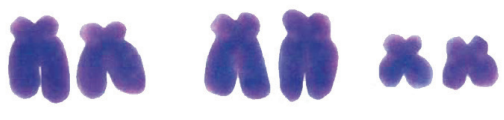

2

3

4
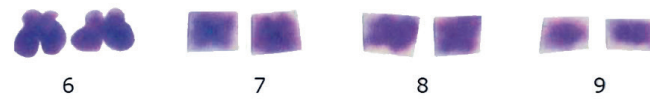

9

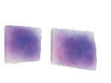

10

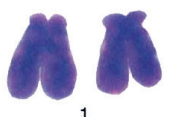

(B)

Figura 3. Cariotipo de Carollia perspicillata del departamento de Córdoba, Colombia. A. Macho $(2 \mathrm{n}=21)$, $21 X Y_{1} Y_{2}$. B. Hembra $(2 n=20), 20 X X$.

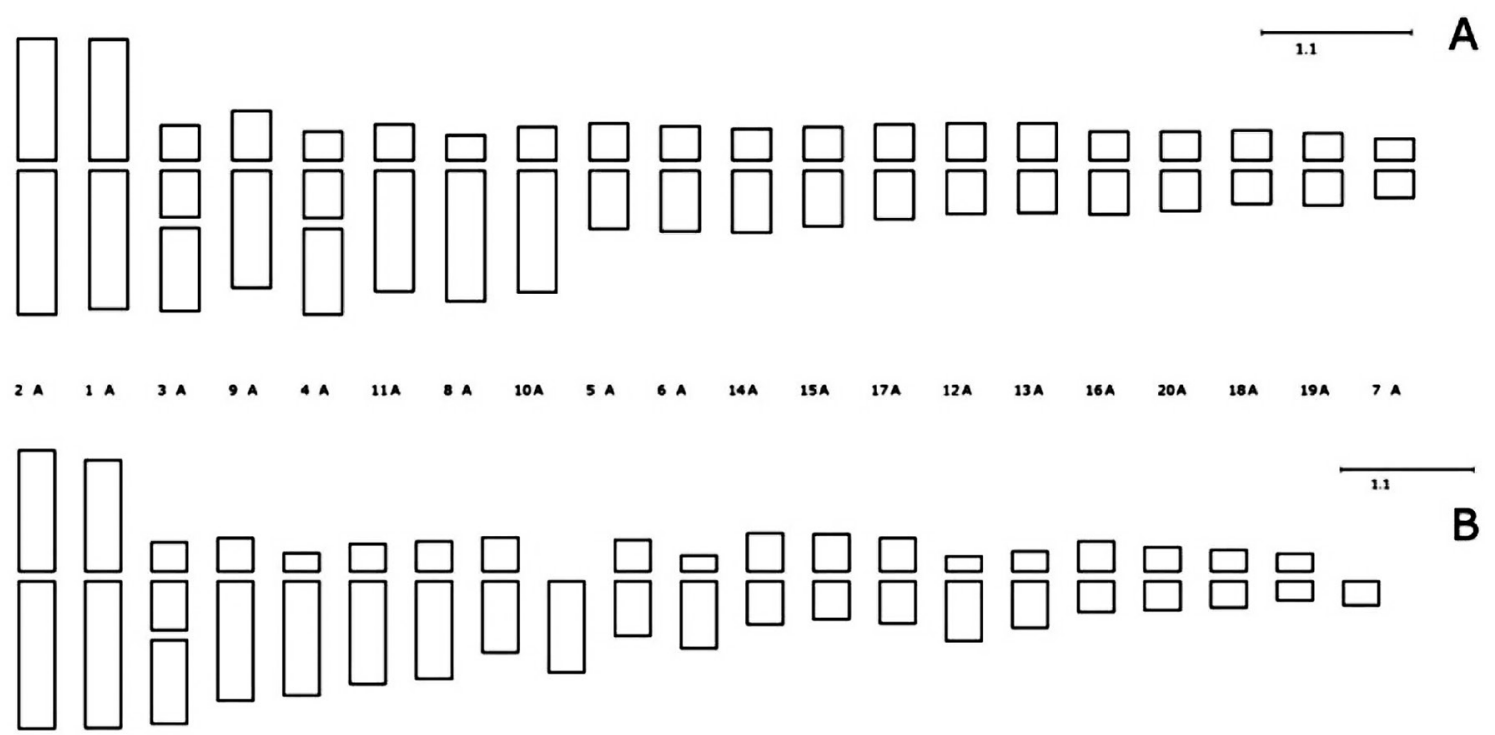

2A 1A 3A GA 5A BA, IA 11A AA 15A 10A IBA 17A 12A OA A 16A 19A 20A 23A 21A 19A

Figura 4. Ideograma de Carollia perspicillata del departamento de Córdoba, Colombia. A. Hembra. B. Macho. Escala $1.1 \mu \mathrm{m}$. 


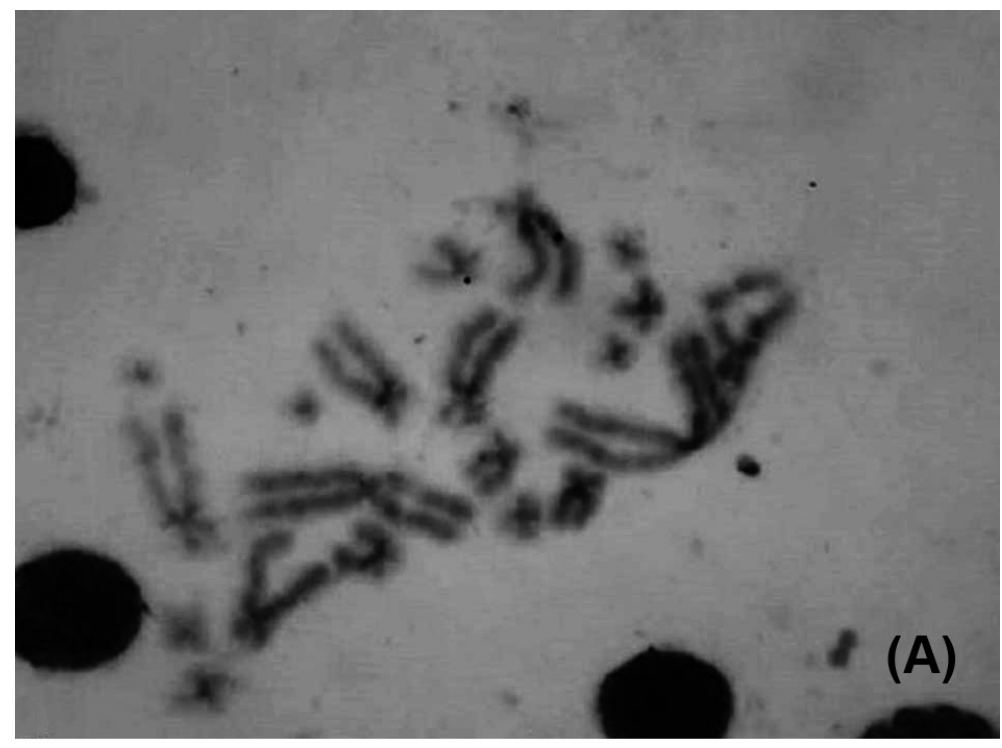

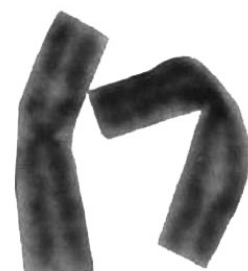

2

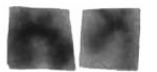

6

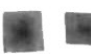

10

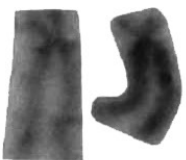

3

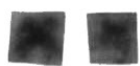

7
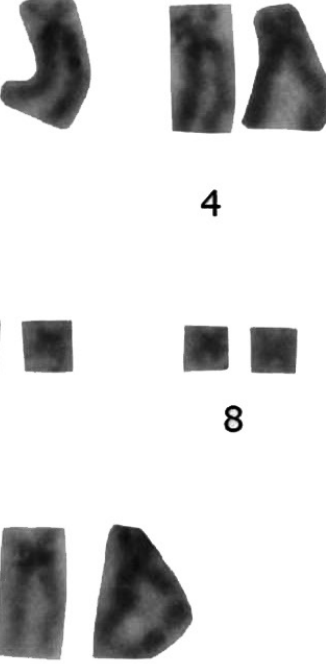

4

5

(B)

Figura 5. Cariotipo de hembra de Carollia perspicillata con Bandeo G.

El cariotipo analizado de hembra de C. perspicillata presentó un par sexual similar a otras hembras de la especie y otras especies del género (Baker \& Bleier 1971, Patton \& Gardner 1971). La fórmula autosómica descrita en los resultados para las hembras es diferente a las mencionadas en la literatura para la especie (Baker 1967, Baker Bleier 1971), pero coincide con una de las tres fórmulas planteadas por Noguera (2008), ya que en la mayoría de las especie del genero Carollia, ocurre esto por las diferencias en las condiciones de preparación del material celular, ya que en algunos casos las variables son incontrolables por trabajar con células mitóticas asincrónicas (Noguera 2008).

Carollia perspicillata, tiene como mecanismo sexual XX/ $\mathrm{XY}_{1} \mathrm{Y}_{2}$ (dimorfismos en los cromosomas sexuales), como también lo presenta la especie $C$. brevicauda según lo descrito por otros autores (Baker 1967, Cloutier \& Thomas 1992, Hsu et al. 1968, Santos \& de Souza 1998), lo que es conocido para el género de murciélagos, Choeronismus (Yonenaga et al. 1969). Dos clases de mecanismos directos pueden ser contemplados para el origen de este sistema; el primero es la fisión céntrica de un cromosoma $Y$ con dos brazos o la translocación autosomal del cromosoma X (Baker 1967, Hsu et al. 1968, Yonenaga et al. 1969).

En muchos quirópteros con un mecanismo sexual simple, el cromosoma $\mathrm{Y}$ es diminuto o es un pequeńo cromosoma acrocéntrico, pero en algunos, (por ejemplo: Sturnira lilium, S. huisi, Chiroderma villosum y especies del género Uroderma) el cromosoma $Y$ marcadamente tiene dos brazos y es la tercera parte del tamaño del cromosoma X (Pinto 2007, Noguera et al. 2009). Para poder explicar el mecanismo sexual de C. perspicillata, también presentado en otras especies del genero Carollia, varios autores como Baker (1967), Hsu et al. (1968), y Yonenaga et al. (1969), han planteado que a partir de una mala división de un cromosoma $Y$ bi-braquial, se pudieron producir múltiples $\mathrm{XY}_{1} \mathrm{Y}_{2}$, contando con las siguientes características al momento del origen: 1) Ambos cromosomas $Y_{1}$ y $Y_{2}$, debieron ser teocéntricos y su tamańo integrado puede ser igual al $Y$ original. 2) Los múltiples cromosomas $\left(\mathrm{XY}_{1} \mathrm{Y}_{2}\right)$, pudieron ser mantenidos 
por quiasma, solo si el emparejamiento de estos fuere cogido en ambos brazos del cromosoma X y Y inicial. 3) No hubo de un todo un cambio cuantitativo y el número de brazos de cromosomas permanecen en ambos sexos. 4) El cromosoma X, mantiene su dimensión inicial.

Por otro lado, Baker (1967), Hsu et al. (1968) y Yonenaga et al. (1969) también sugieren mecanismos de origen basados en el intercambio autosoma-gonosoma $\mathrm{X}$, lo que pudo significar que:

1. El cromosoma Y inicial podría estar sin cambios mientras él Y adicional podría corresponder en estructura y contenido genético a un cromosoma autosómico del conjunto básico.

2. Algunas perdidas de material céntrico, terminal, procentrico, podrían estar involucrados y así por lo menos tener la probabilidad del éxito.

3. El número de pares autosómicos podrían estar reducidos a uno.

4. El tamaño del cromosoma X podría probablemente haber incrementado por la longitud de acercamiento del segmento autosomal traslocado.

Por tales motivos, es factible creer que el mecanismo múltiple sexual de los murciélagos ha tenido una historia polifilética que no puede ser claramente definida (Baker 1967, Hsu et al. 1968).

En C. perspicillata los brazos cortos de los cromosomas $\mathrm{Y}_{1}$ y $Y_{2}$ no pueden ser detectados, sin embargo, la unión de la longitud de estos cromosomas en relación a la del cromosoma $\mathrm{X}$, superaría la medida de un cromosoma $\mathrm{Y}$ de las especies $\mathrm{XY}$ conocidas (no trivalentes); por otro lado, el cromosoma $Y_{2}$ se asemeja al cromosoma $\mathrm{Y}$ pequeño de las especies $\mathrm{XY}$ que se caracterizan por tener un Y diminuto. Lo anterior, sería conveniente, no obstante, en cualquiera de los modos de origen del mecanismo sexual propuesto, no se esperaría que en los cromosomas involucrados $\left(\mathrm{Y}_{1}, \mathrm{Y}_{2}\right)$ se conserve completamente la relación de la meiosis en individuos machos $(\mathrm{XY})$ que ha sido descrita por (Bovey R. 1949). Estudios en las especies que tienen $X_{1} Y_{2}$ (Yonenaga et al. 1969) han revelado un mecanismo sexual trivalente en machos, como en el caso de C. perspicillata, corroborado en los resultados.

El patrón de bandeo G para C. perspicillata es similar al de C. brevicauda y los cromosomas sexuales han demostrado ser translocaciones del cromosoma X original, como lo muestran estudios similares realizados en México (Sotck 1975).

Algunos autores (Baker 1967, Hsu et al. 1968) han usado la terminología de "sistema múltiple de cromosomas sexuales" el cual ha sido confuso, en la descripción de cariotipos como el de Carollia perspicillata. Los patrones de bandeo permiten una identificación positiva de segmentos traslocados, que puede ser más conveniente para indicarlos como translocaciones en lugar de mecanismo múltiple de cromosomas sexuales (Sotck 1975).

Con este trabajo se incrementa la representatividad geográfica de los estudios citogenéticos de C. perspicillata para la costa norte de Colombia. Las características cromosómicas mostradas para esta especie, permiten identificar citogenéticamente divergencias interespecíficas con las otras especies del género Carollia, proporcionando una herramienta adecuada para los problemas taxonómicos existentes.

\section{Agradecimientos}

Al Programa de Estudios en Infecciones y Salud Tropical PEIST, al Grupo de Investigaciones Microbiológicas y Biomédicas de Córdoba GIMBIC, al Grupo de Investigación Biomédica y Biología Molecular de la Universidad del Sinú, por el soporte prestado en la fase de laboratorio. A Andrés Jattin y Mayra Ballestas por su colaboración en el desarrollo de las técnicas de laboratorio y a Jairo Martínez por su colaboración en la colecta de especímenes

\section{Literatura citada}

Baker R. J., \& J. Bleier. 1971. Karyotypes of bats of the subfamily Carolliinae (Mammalia; Phyllostomatidae) and their evolutionary implications. Experientia 27: 220-222. doi: https:// doi.org/10.1007/BF02145907

Baker R. J. 1967. Karyotypes of bats of the family Phyllostomidae and their taxonomic implications. The Southwestern Naturalist 12: 407-428. doi: 10.2307/3669608

Baker R. J., M. J. Hamilton, \& D. A. Parish. 2003. Preparations of mammalian karyotypes under field conditions. Occasional Papers, Museum of Texas Tech University 228: 1-8.

Baker R. J., \& M. B. Qumsiyeh. 1988. Methods in chiropteran mitotic chromosomal studies. In: Kunz T. H., ed. Ecological and behavioral methods for the study of bats. Washington, DC: The Smithsonian Institution Press. pp. 425-435.

Ballesteros J., \& J. Racero-Casarrubia. 2012. Murciélagos del área urbana en la ciudad de Montería, Córdoba - Colombia. Revista MVZ Córdoba 17: 3193-9.

Fleming T. H. 1982. Foraging strategies of plant visiting bats. In: Kunz T.H, ed. Ecology of Bats. New York: Plenum Press. pp. 287-325. doi: https://doi.org/10.1007/978-1-46133421-7_8

Bovey R. 1949. Les chromosomes des Chiropteres et des Insectivores. Rev Suisse Zool 56: 371-460. doi: 10.1080/00087114.1969.10796320

Calonge B., I. M. Velaa-Vargas, \& J. Pérez-Torres. 2010. Murciélagos asociados a una finca ganadera en Córdoba (Colombia). Revista MVZ Córdoba 15: 1938-43.

Castro-Luna, A., \& J. Galindo-González. 2012. Seed dispersal by Phyllostomid bats in two contrasting vegetation types in a mesoamerican reserve. Acta Chiropterologica 14: 133-42. doi: https://doi.org/10.3161/150811012X654349

Chacón J., E. Humanez-López, \& J. A. Martínez. 2015. Murciélagos asociados con un área de restauración ecológica en el Alto Sinú, departamento de Córdoba, Colombia. Revista Biodiversidad Neotropical 5: 120-3. doi: http://dx.doi. org/10.18636/bioneotropical.v5i2.207

Cloutier D., \& D. Thomas. 1992. Carollia perspicillata. Mammalian species 417: 1-9. doi: https://doi.org/10.2307/3504157

Gardner A. L. (ed.). 2008. Mammals of South America, volume 1: marsupials, xenarthrans, shrews, and bats. Chicago, EE.UU: The University of Chicago Press. 690 pp.

Harrison R. D. 2005. Figs and the Diversity of Tropical Rainforests. Bioscience 55: 1053-1064. doi: https://doi. org/10.1641/0006-3568(2005)055[1053:FATDOT]2.0 .CO;2

Hoffmann F. G., \& R. J. Baker. 2003. Comparative phylogeography of shorttailed bats (Carollia: Phyllostomidae). Mol Ecol. 12: 3403-14. doi: 10.1046/j.1365-294X.2003.02009.x

Hsu T. C., R. J. Baker, \& T. Utakoji. 1968. The multiple sex chromosome system of American leaf-nosed bats (Chiroptera, Phyllostomidae). Cytogenetics 7: 27-38.

Jac M., \& J. Scheres. 1972. Identification of two Robertsonian translocations with a Giemsa banding technique. Hum Genet. 15: 253-6. doi: https://doi.org/10.1007/BF00702361

Levan A., K. Fredga, \& A. A. Sandberg. 1964. Nomenclature for centromeric position on chromosomes. Hereditas 52: 201-220. doi: 10.1111/j.1601-5223.1964.tb01953.x

Linares O. 1998. Mamíferos de Venezuela. Caracas: Sociedad Conservacionista Audobun de Venezuela. 691 pp. 
MacLellan L., \& K. Koopman. 2008. Subfamily Carolliinae. In: Gardner A. L., ed. Mammals of South America: Marsupials, xenarthrans, shrews and bats. Chicago, EE.UU: The University of Chicago Press. pp. 210.

Medellín R. A., M. Equihua, \& M. A. Amin. 2000. Bat diversity and abundance as indicatos of disturbance in neotropical rainforest. Conservation Biology 14:1666-1675. doi: 10.1111/j.1523-1739.2000.99068.x

Muñoz J., C. A. Cuartas-Calle, \& M. González. 2004. Se describe una nueva especie de murciélago del género Carollia Gray, 1838 (Chiroptera: Phyllostomidae) de Colombia. Actualidades Biológicas 26:80-90.

Muscarella R., \& T. H. Fleming. 2007. The role of frugivorous bats in tropical forest succession. Biol Rev. 82: 573-90. doi: 10.1111/j.1469-185X.2007.00026.x

Noguera E. A. 2008. Cariología comparada de Carollia perspicillata y Carollia brevicauda (Chiroptera: Phyllostomidae: Carolliinae) en la Provincia Biogeográfica Norandina de Nariño. Trabajo de grado, Biología. Facultad de Ciencias Exactas y Naturales Universidad de Nariño. Pasto, 116 pp.

Noguera E. A., H. E. Ramírez-Chaves, \& S. Montenegro. 2009. Primer registro del cariotipo de Sturnira luisi Davis, 1980. Chiroptera Neotropical 15: 477-483.

Noguera-Urbano E. A., \& S. Muńoz-Montenegro. 2014. Un cariotipo del murciélago sedoso de cola corta (Carollia brevicauda [Schinz,1821], Chiroptera: Phyllostomidae) de los Andes de Colombia. Therya 5: 559-66. doi: http://dx.doi. org/10.12933/therya-14-145

Patton J. L. 1967. Chromosome studies of certain pocket mice genus Perognathus (Rodentia: Heteromyidae). Journal of Mammalogy 48: 27-37. doi: 10.2307/1378167

Patton J. L., \& A. L. Gardner. 1971. Parallel evolution of multiple sex-chromosome systems in the phyllostomatid bats, Carollia and Choeroniscus. Cellular and Molecular Life Sciences 27: 105-106. doi: https://doi.org/10.1007/BF02137768

Peńuela-Salgado M., \& J. Pérez-Torres. 2015. Environmental and spatial characteristics that affect roost use by Seba's shorttailed bat (Carollia perspicillata) in a Colombian cave. Journal of Cave and Karst Studies 77: 160-164. doi: 10.4311/2015LSC0105

Pinto M. M. 2007. Utilização de marcadores citogenéticos na análise comparativa dos grandes Artibeus (Phyllostomidae, chiroptera), avaliando estruturas conservadas e sítios espécieespecíficos. Dissertação Mestre em Genética, Facultad de Universidade Federal de Pernambuco. Recife, Brasil. 81 pp.

Racero-Casarrubia J., \& J. González-Maya. 2014. Inventario preliminar y uso de mamíferos silvestres por comunidades campesinas del sector oriental del cerro Murrucucú, municipio de Tierralta, Córdoba, Colombia. Mammalogy Notes 1: 25-28.

Racero-Casarrubia J., J. Ballesteros-Correa, \& J. Pérez-Torres. 2015. Mamíferos del departamento de Córdoba-Colombia: historia y estado de conservación. Biota Colombiana 16: 69-89.
Ramírez-Chaves H. E., \& E. Noguera. 2010. Lista preliminar de los mamíferos (Mammalia: Theria) del departamento de Narińo, Colombia. Biota Colombiana 11: 117-40.

Santos N. \& M. J. Souza. 1998. Characterization of the constitutive heterochromatin of Carollia perspicillata (Phyllostomidae, Chiroptera) using the base specific fluorochromes, CMA (3) (GC) and DAPI (AT). Caryologia 51: 51-60. doi: https:// doi.org/10.1080/00087114.1998.10589119

Simmons N. B. 2005. Order Chiroptera. In: Wilson D.E. \& Reeder D.M., eds. Mammal Species of the World: a taxonomic and geographic reference. 3rd ed., Vol 1. Baltimore, Maryland: Johns Hopkins University Press. pp. 312-529.

Solari S., \& R. J. Baker. 2006. Mitochondrial DNA sequence, karyotypic, and morphological variation in the Carollia castanea species complex (Chiroptera: Phyllostomidae) with description of a new species. Occasional Papers, Museum of Texas Tech University 254: 1-16.

Solari S., \& V. Martínez-Arias. 2014. Cambios recientes en la sistemática y taxonomía de murciélagos Neotropicales (Mammalia: Chiroptera). Therya 5: 167-96. doi: http://dx.doi. org/10.12933/therya-14-180

Sotck A. 1975. Chromosome banding pattern homology and its phylogenetic implications in the bat genera Carollia and Choeroniscus. Cytogenet cell Genet. 14:34-41. doi: https:// doi.org/10.1159/000130317

Sotero-Caio C. G., M. Volleth, L. S. Gollahon, B. Fu, W. Cheng, B. L. Ng, F. Yang, \& R. J. Baker. 2013. Chromosomal evolution among leaf-nosed nectarivorous bats-evidence from cross-species chromosome painting (Phyllostomidae, Chiroptera). BMC Evolutionary Biology 13: 276. doi: https://doi.org/10.1186/1471-2148-13-276

Suárez-Villota E. Y., J. Racero-Casarrubia, G. Guevara, \& J. Ballesteros. 2009. Evaluación ecológica rápida de los quirópteros del parque ecológico de Montelíbano, Córdoba, Colombia. Tropical Conservation Science 2: 437-49. doi: https://doi. org/10.1177/194008290900200405

Timm R. M. \& R. K. LaVal. 1998. A field key to the bats of Costa Rica. Occasional Publication Series, University of Kansas Center of Latin American Studies 22: 1-30.

Vela-Vargas I. M., \& J. Pérez-Torres. 2012. Murciélagos asociados a remanentes de bosque seco tropical en un sistema de ganadería extensiva (Colombia). Chiroptera Neotropical 18: 1089-100.

Yonenaga Y., O. Frota-Pessoa, \& K. R. Lewis. 1969. Karyotypes of seven species of Brazilian bats. Caryologia 22: 63-79. doi: $10.1080 / 00087114.1969 .10796325$

Zurc D., \& P. M. Velasco. 2010. Análisis morfológico y morfométrico de Carollia colombiana Cuartas et al. 2001 y C. monohernandezi Muñoz et al. 2004 (Phyllostomidae: Carollinae) en Colombia. Chiroptera Neotropical 16: 567-572. 